UT-599

March 1992

\title{
BRST-Fixed Points and Topological Conformal Symmetry
}

\author{
Toshio Nakatsu and Yuji Sugawara \\ Department of Physics, University of Tokyo \\ Bunkyo-ku,Tokyo 113,Japan
}

\begin{abstract}
We study the twisted version of the supersymmetric $G / T=S U(n) / U(1)^{\otimes(n-1)}$ gauged Wess-Zumino-Witten model. By studying its fixed points under BRST transformation this model is shown to be reduced to a simple topological field theory, that is, the topological matter system in the K.Li's theory of 2 dimensional gravity for the case of $n=2$, and its generalization for $n \geq 3$.
\end{abstract}


The idea of BRST-fixed points has been introduced into the study of some topological field theories (TFTs) with BRST symmetries [1]. It has been pointed out [1], [2] that the path-integration of BRST-invariant field theories is reduced to an integral over fixed point sets of BRST transformation and the original TFT may be reduced to a much simple one. In this article we will show that the twisted version of the supersymmetric $G / T=S U(n) / U(1)^{\otimes(n-1)}$ gauged Wess-Zumino-Witten (WZW) model is reduced to a simple supersymmetric Coulomb gas system. This system coincides with the topological matter system in the K.Li's theory of 2 dimensional gravity for the case of $n=2$, and its generalization for $n \geq 3$. We argue that the reduction to fixed points in operator language is given by the cancellation mechanism between bosonic and fermionic ghosts known in the theory of Hamiltonian reduction (quartet mechanism).

The topological conformal model [3] which is obtainable by twisting the $\mathrm{N}=2$ superconformal model based on the flag manifold $G / T=S U(n) / U(1)^{\otimes(n-1)}$ [4] was known to be described as two dimensional Lagrangian field theory [1]. The action of them is given by;

$$
I(g, \chi, \psi, A)=k S_{G}(g, A)+S_{\chi \psi}(\chi, \bar{\chi}, \psi, \bar{\psi}, A)
$$

The bosonic part of the action $S_{G}$ (1) is that of the $G=S U(n)$ WZW model gauged by the Cartan subgroup $T=U(1)^{\otimes(n-1)}$, which can be obtained by adding the following gauge interaction to $S_{G}^{W Z W}(g)$, the action of an ungauged $G$ WZW model;

$$
\begin{aligned}
& k S_{G}(g, A) \\
& =k S_{G}^{W Z W}(g)+\frac{i k}{2 \pi} \int_{\Sigma} d^{2} z \operatorname{Tr}\left\{-g^{-1} \bar{\partial} g A_{z}+A_{\bar{z}} \partial g g^{-1}-A_{\bar{z}} g A_{z} g^{-1}+A_{\bar{z}} A_{z}\right\}
\end{aligned}
$$

where $g$ is a chiral field on a Riemann surface $\Sigma$, taking its value in $G$, and $A=A_{z} d z+A_{\bar{z}} d \bar{z}$ 円 is $T=U(1)^{\otimes(n-1)}$ gauge field on $\Sigma$. The fermionic part of the action (四) is ;

$$
S_{\chi \psi}(\chi, \bar{\chi}, \psi, \bar{\psi}, A)=\frac{1}{2 \pi i} \int_{\Sigma} d^{2} z \operatorname{Tr}\left\{\chi_{z} \bar{\partial}_{A_{\bar{z}}} \psi+\bar{\chi}_{\bar{z}} \partial_{A_{z}} \bar{\psi}\right\}
$$

\footnotetext{
${ }^{1} A_{z}, A_{\bar{z}}$ satisfy $A_{z}^{\dagger}=-A_{\bar{z}}$.
} 
The fermionic fields $\chi, \bar{\chi}, \psi, \bar{\psi}$ are introduced in the following way: The ghost fields $\psi, \bar{\psi}$ behave as $(0,0)$-forms on $\Sigma$, taking their values in $m_{+}, m_{-}$respectively. Here $m_{ \pm}$are the components of the Cartan-Weyl decomposition of $s u(n): s u(n)=t \oplus m_{+} \oplus m_{-}$. The antighost fields $\chi=\chi_{z} d z, \bar{\chi}=\bar{\chi}_{\bar{z}} d \bar{z}$ behave as $(1,0),(0,1)$-forms on $\Sigma$, taking their values in $m_{-}, m_{+}$respectively. Note that the space $m_{+} \oplus m_{-}$can be regarded as the (complexified) tangent space of the flag manifold $G / T=S U(n) / U(1)^{\otimes(n-1)}$. Thus we will call these fermionic fields as coset ghosts. The covariant derivative $d_{A}=\partial_{A_{z}}+\bar{\partial}_{A_{\bar{z}}}$ is defined via the adjoint action of the Cartan subgroup $T$ on the flag manifold; $d_{A}=d+[A, \cdot]$.

The action (1) has the fermionic symmetry of the following type 9 ;

$$
\begin{aligned}
\delta_{G / T} g=\psi g, & \bar{\delta}_{G / T} g=-g \bar{\psi}, \\
\delta_{G / T} \chi_{z, \alpha}=\operatorname{Tr} e_{\alpha}\left(k \partial_{A_{z}} g g^{-1}+\left[\chi_{z}, \psi\right]\right), & \bar{\delta}_{G / T} \bar{\chi}_{\bar{z}}^{\alpha}=\operatorname{Tr} e^{\alpha}\left(-k g^{-1} \bar{\partial}_{A_{\bar{z}}} g+\left[\bar{\chi}_{\bar{z}}, \bar{\psi}\right]\right), \\
\delta_{G / T} \psi=\frac{1}{2}[\psi, \psi], & \bar{\delta}_{G / T} \bar{\psi}=\frac{1}{2}[\bar{\psi}, \bar{\psi}] .
\end{aligned}
$$

Contributions other than the above described vanish. Note that the above BRST-like symmetry is that obtained by twisting the $\mathrm{N}=2$ superconformal symmetry realized by the Kazama-Suzuki formalism [4]. We will call it $G / T$-BRST symmetry.

The path-integral quantization of the model (11) is performed along the formalism developed in [5], [6], by utilizing the concept of "chiral gauge transformation", that is, complex gauge transformation defined for any $T^{c}$-valued function. Under the chiral gauge transformation, the bosonic part of the action (10) satisfies the "Polyakov-Wiegmann identity";

$$
S_{G}\left({ }^{\Omega} g,{ }^{\Omega} A\right)=S_{G}(g, A)-S_{G}\left(\Omega^{\dagger} \Omega, A\right),
$$

where $\Omega$ is $T^{c}=\left(U(1)^{c}\right)^{\otimes(n-1)}$-valued, and

$$
\begin{aligned}
& { }^{\Omega} g=\Omega^{\dagger-1} g \Omega^{-1} \\
& \left({ }^{\Omega} A\right)_{z}=-\partial_{z} \Omega \Omega^{-1}+A_{z} .
\end{aligned}
$$

The "Polyakov-Wiegmann identity" tells us that the bosonic part (2) has the gauge invariance for the vectorial direction, that is, the $T=U(1)^{\otimes(n-1)}$ gauge transformation. But

\footnotetext{
${ }^{2}$ We parametrize the coset (anti-)ghosts such as $\psi=\sum_{\alpha \in \Delta_{+}} \psi^{\alpha} e_{\alpha}\left(\chi_{z}=\sum_{\alpha \in \Delta_{+}} \chi_{z \alpha} e^{\alpha}\right)$. Here $\Delta_{+}$ is the set of the positive roots of $s u(n)$, and $\left\{e_{\alpha}\right\}_{\alpha \in \Delta_{+}},\left\{e^{\alpha}\right\}_{\alpha \in \Delta_{+}}$are the Cartan-Weyl basis of $m_{+}, m_{-}$ respectively.
} 
when $\Omega$ is $T^{c} / T$-valued (i.e the axial direction), The theory suffers the chiral anomaly at the classical level. Of course the fermionic part (3) has no anomaly at the classical level. But, at the quantum level, their determinant behaves anomalously for the axial direction of the chiral gauge transformation.

Now suppose we parametrize the gauge field $A$ as

$$
A_{z}=-\partial_{z} \Omega \Omega^{-1}
$$

where $\Omega=e^{X} e^{i Y}$ is $T^{c}=\left(U(1)^{c}\right)^{\otimes(n-1)}$-valued $\left(e^{X}, e^{i Y}\right.$ are $T^{c} / T, T$-valued respectively). With this parametrization (7) we can perform the path-integration of the model. Note that, while $\int D Y$, being simply the gauge volume, can be dropped without any trouble, we cannot drop $\int D X$ because of the chiral anomaly. After some calculations of the anomalies we obtain the following relation [7], [8];

$$
\begin{aligned}
& \int \frac{D A D g D(\chi, \bar{\chi}, \psi, \bar{\psi})}{\text { gauge volume }} e^{-I(g, \chi, \psi, A)} \\
& =\int D X D g D(\chi, \bar{\chi}, \psi, \bar{\psi}) D(\zeta, \bar{\zeta}, \xi, \bar{\xi}) e^{-I^{g . f}(g, X, \chi, \psi, \zeta, \xi)}
\end{aligned}
$$

where the gauge fixed action $I^{g \cdot f}$ is given by ;

$$
\begin{aligned}
& I^{g . f}(g, \chi, \bar{\chi}, \psi, \bar{\psi}, \zeta, \bar{\zeta}, \xi, \bar{\xi}) \\
& \quad=k S_{G}^{W Z W}(g)+S_{X}(X)+S_{\chi \psi}(\chi, \bar{\chi}, \psi, \bar{\psi})+S_{\zeta \xi}(\zeta, \bar{\zeta}, \xi, \bar{\xi})
\end{aligned}
$$

In the above $X=\left(X_{1}, \cdots, X_{n-1}\right)$ are non-compact free bosons originating from the gauge field $A$ with the action?

$$
S_{X}(X)=\frac{1}{2 \pi i} \int_{\Sigma} d^{2} z \partial_{z} X \cdot \bar{\partial}_{\bar{z}} X+\frac{1}{2 \pi} \int_{\Sigma} \sqrt{2} \alpha_{-} \rho \cdot X R
$$

$\zeta=\left(\zeta_{1}, \cdots, \zeta_{n-1}\right), \xi=\left(\xi_{1}, \cdots, \xi_{n-1}\right)$ are spin $(1,0)$ ghost systems representating the Jacobian of the parametrization (17). We will call these (anti-)ghost fields $\xi, \bar{\xi}\left(\zeta_{z}, \bar{\zeta}_{\bar{z}}\right)$ as $T^{c}$-ghosts. Their action is

$$
S_{\zeta \xi}(\zeta, \bar{\zeta}, \xi, \bar{\xi})=\frac{1}{2 \pi i} \int_{\Sigma} d^{2} z \zeta_{z} \cdot \bar{\partial}_{\bar{z}} \xi+\bar{\zeta}_{\bar{z}} \cdot \partial_{z} \bar{\xi}
$$

\footnotetext{
${ }^{3}$ In what follows, we set $\rho$ the Weyl vector of $s u(n)$ and define $\alpha_{+}=\sqrt{k+n}, \alpha_{-}=-\frac{1}{\sqrt{k+n}}$.
} 
At this stage the quantization of the model is straightforward, since everything is expressed either in free fields, or in terms of an ungauged $G$ WZW model. First we note that, in order to extract the physical degrees of freedom from our total system, one needs to use the BRST symmetries of the combined system (9). The gauge fixing for the chiral gauge transformation (6) should be characterized by the nilpotent BRST charge $Q_{T^{c}}$ which generates $T^{c}$-BRST transformation;

$$
\begin{aligned}
Q_{T^{c}} & =\frac{1}{2 \pi i} \int G_{T^{c}}^{+}, \\
G_{T^{c}}^{+} & =-\alpha_{-}\left(\xi \cdot H^{\text {total }}-\sqrt{2} \rho \cdot \partial \xi\right),
\end{aligned}
$$

where $H^{\text {total }}$ is an anomaly free $t$-current of the combined system ;

$$
H^{\text {total }}=\hat{H}+H_{X}
$$

Here $\hat{H}$ is the current associated with the $T=U(1)^{\otimes(n-1)}$ gauge symmetry, which is composed of $H^{W Z W}$, the Cartan current of the $S U(n)$ WZW model and $H_{\chi \psi}=\frac{1}{\sqrt{2}} \sum_{\alpha \in \Delta_{+}} \alpha \chi_{\alpha} \psi^{\alpha}$, that of the coset ghosts f. $H_{X}=\alpha_{+} \partial X$ is the current of the gauge field 0 .

As we described before, the nilpotent BRST charge $Q_{G / T}$ which generates the $G / T$ BRST transformation (幽) can be constructed from one of the supersymmetry operators realized by the Kazama-Suzuki formalism [四];

$$
\begin{aligned}
Q_{G / T} & =\frac{1}{2 \pi i} \int G_{G / T}^{+} \\
G_{G / T}^{+} & =-\alpha_{-} \sum_{\alpha \in \Delta_{+}} \psi^{\alpha}\left(J_{\alpha}^{W Z W}-\sum_{\beta \in \Delta_{+}, \alpha<\beta} \psi^{\beta} \chi_{\alpha+\beta}\right) .
\end{aligned}
$$

Note that the BRST currents $G_{T^{c}}^{+}(12)$ and $G_{G / T}^{+}$(14) anticommute each other. Then the coresponding BRST charges $Q_{T^{c}}, Q_{G / T}$ anticommute. The physical observables can be characterized as the cohomology classes of the double complex, whose differential is given by the total BRST charge $Q^{\text {total }}=Q_{T^{c}}+Q_{G / T}$. In what follows, assuming the degeneration of the corresponding spectral sequence, we will study this cohomological problem by taking cohomology first by $Q_{G / T}$ and then by $Q_{T^{c}}$.

The total energy-momentum (EM) tensor of the combined system (9) is easily derived;

$$
T^{t o t}=T_{W Z W}+T_{\chi \psi}+T_{X}+T_{\zeta \xi}
$$

\footnotetext{
${ }^{4}$ They satisfy the OPEs: $H_{i}^{W Z W}(z) H_{j}^{W Z W}(w) \sim \frac{\frac{k}{2} \delta_{i j}}{(z-w)^{2}}, H_{\chi \psi, i}(z) H_{\chi \psi, j}(w) \sim \frac{\frac{n}{2} \delta_{i j}}{(z-w)^{2}}$.

${ }^{5} H_{X, i}(z) H_{X, j}(w) \sim \frac{-\frac{k+n}{2} \delta_{i j}}{(z-w)^{2}}$.
} 
where $T_{W Z W}$ is the EM tensor of the $S U(n)$ WZW model, and $T_{X}, T_{\chi \psi}, T_{\zeta \xi}$ are those of the gauge field, the coset ghosts, the $T^{c}$-ghosts respectively. Their explicit forms are given by ;

$$
\begin{aligned}
& T_{X}=-\partial X \cdot \partial X+\sqrt{2} \alpha_{-} \rho \cdot \partial^{2} X, \\
& T_{\chi \psi}=-\sum_{\alpha \in \Delta_{+}} \chi_{\alpha} \partial \psi^{\alpha}, \quad T_{\zeta \xi}=-\zeta_{z} \cdot \partial \xi
\end{aligned}
$$

From the above explicit forms we can see that $T^{\text {tot }}$ has vanishing central charge. So the model is a TFT. Moreover $T^{\text {tot }}$ can be written as the following BRST exact form;

$$
T^{t o t}=\left\{Q^{t o t}, G_{G / T}^{-}+G_{T^{c}}^{-}\right\},
$$

where $G_{G / T}^{-}$is the spin 2 fermionic current constructed from the other supersymmetry operator realized by the Kazama-Suzuki formalism, and $G_{T^{c}}$ is also a spin 2 fermionic current. Its explicit form is;

$$
G_{T^{c}}^{-}=-\alpha_{-}\left(\zeta_{z} \cdot\left(\hat{H}-H_{X}\right)-\sqrt{2} \rho \cdot \partial \zeta_{z}\right)
$$

We observe that the BRST currents $G_{G / T}^{+}, G_{T^{c}}^{+}$anti-commute with $G_{T^{c}}^{-}, G_{G / T}^{-}$respectively. Hence we can factorize the BRST-exact form (17) into two commuting pieces;

$$
T^{t o t}=T_{G / T}+T_{T^{c}}
$$

where we set

$$
\begin{aligned}
T_{G / T} & =\left\{Q_{G / T}, G_{G / T}^{-}\right\} \\
& =T_{W Z W}+T_{\chi \psi}-\frac{1}{k+n} \hat{H} \cdot \hat{H}+\frac{\sqrt{2}}{k+n} \rho \cdot \partial \hat{H} \\
T_{T^{c}} & =\left\{Q_{T^{c}}, G_{T^{c}}^{-}\right\} \\
& =T_{X}+T_{\xi \zeta}+\frac{1}{k+n} \hat{H} \cdot \hat{H}-\frac{\sqrt{2}}{k+n} \rho \cdot \partial \hat{H} .
\end{aligned}
$$

Note that $\left\{T_{G / T}, G_{G / T}^{ \pm}, J_{K S}\right\}$ generate the topological conformal algebra (TCA) obtained by twisting the Kazama-Suzuki model for the flag manifold $G / T=S U(n) / U(1)^{\otimes(n-1)}$ (central charge $\left.C_{K S}=\frac{3}{2} n(n-1)-\frac{12 \rho^{2}}{k+n}\right)$, with the definition of the $U(1)$ current,

$$
J_{K S}=\frac{2 \sqrt{2}}{k+n} \rho \cdot \hat{H}+N_{\chi \psi},
$$

$$
{ }^{6} G_{G / T}^{+}(z) G_{T^{c}}^{-}(w) \sim G_{T^{c}}^{+}(z) G_{G / T}^{-}(w) \sim \text { regular at } z=w .
$$


where $N_{\chi \psi}$ is the ghost number current for the coset ghosts $\chi_{z}, \psi$.

Suppose we firstly take cohomology by $Q_{G / T}$. Then the reduced system (with respect to $Q_{G / T}$ ) will be characterized by the EM tensor $T_{T^{c}}$. Observing that $T_{T^{c}}$ has vanishing central charge and can be written as the BRST-exact form $T_{T^{c}}=\left\{Q_{T^{c}}, G_{T^{c}}^{-}\right\}$, we can ask if it is possible to characterize this reduced system by an appropriate TCA. There exists such a TCA. Namely we can see that $\left\{T_{T^{c}}, G_{T^{c}}^{ \pm}, J_{T^{c}}\right\}$ generate a TCA with a $U(1)$ current;

$$
J_{T^{c}}=-\frac{2 \sqrt{2}}{k+n} \rho \cdot H_{X}+N_{\zeta \xi}
$$

where $N_{\zeta \xi}$ is the ghost number current for the $T^{c}$-ghosts $\zeta, \xi$. From the OPE among this $U(1)$ current, we see the above TCA is obtained by twisting a $\mathrm{N}=2$ superconformal algebra with central charge $C_{T^{c}}=3(n-1)-\frac{12 \rho^{2}}{k+n}$.

Let us now connect our analysis to those using path-integral formulation. It has been pointed out in [1] that the path-integration in BRST invariant theories is actually reduced to the integral over the fixed point sets under BRST transformation. We conjecture that, in our case when the BRST operator is identified as $Q_{G / T}$ ([4]), the second piece in ([19) describes the theory reduced on to the BRST fixed points.

In order to discuss this reduction, it is convenient to utilize the free field realization of the $S U(n)$ WZW model (c.f [9]). Introducing compact free bosons $\phi=\left(\phi_{1}, \cdots, \phi_{n-1}\right)$ and bosonic $\left(\beta_{\alpha}, \gamma^{\alpha}\right)$ systems $\left(\alpha \in \Delta_{+}\right)$, the $S U(n)$ WZW model may be described by the action;

$$
k S_{G}^{W Z W}(\phi, \beta, \bar{\beta}, \gamma, \bar{\gamma})=S_{\phi}(\phi)+S_{\beta \gamma}(\beta, \bar{\beta}, \gamma, \bar{\gamma})
$$

where

$$
\begin{aligned}
& S_{\phi}(\phi)=\frac{1}{2 \pi i} \int_{\Sigma} d^{2} z \partial_{z} \phi \cdot \bar{\partial}_{\bar{z}} \phi+\frac{i}{2 \pi} \int_{\Sigma} \sqrt{2} \alpha_{-} \rho \cdot \phi R \\
& S_{\beta \gamma}(\beta, \bar{\beta}, \gamma, \bar{\gamma})=\frac{-1}{2 \pi i} \int_{\Sigma} d^{2} z \sum_{\alpha \in \Delta_{+}}\left(\beta_{z, \alpha} \bar{\partial} \gamma^{\alpha}+\bar{\beta}_{\bar{z}}^{\alpha} \partial \bar{\gamma}_{\alpha}\right)
\end{aligned}
$$

Note that the BRST current $G_{G / T}^{+}(14)$ is essentially the same as that appears in the study of the quantum Hamiltonian reduction àla Drinfeld-Sokolov [10], [1]. We can prove that 
the bosonic ghosts $\left(\beta_{\alpha}, \gamma^{\alpha}\right)_{\alpha \in \Delta_{+}}$and the coset ghosts $\left(\chi_{\alpha}, \psi^{\alpha}\right)_{\alpha \in \Delta_{+}}$form a pair of the $G / T$ BRST doublets (c.f [10 [11]), and so these fields decouple from the theory (the "quartet confinements"). Especially the following relation holds;

$$
S_{\beta \gamma}(\beta, \bar{\beta}, \gamma, \bar{\gamma})+S_{\chi \psi}(\chi, \bar{\chi}, \psi, \bar{\psi})=\left\{Q_{G / T}+\bar{Q}_{G / T}, V\right\}
$$

where $V$ is an appropriate functional. Strictly speaking we have checked the above relation only in the case of $n=2$. But, taking account of the results obtained in [11], it should hold in general.

The fixed points of the $G / T$-BRST transformation (4) is easily derived because the current $J^{W Z W}$ which appears in the definition of the BRST current $G_{G / T}^{+}$(14) can be realized in terms of the bosonic ghosts $\left(\beta_{\alpha}, \gamma^{\alpha}\right)_{\alpha \in \Delta_{+}}$. Taking also the anti-holomorphic part into account, these fixed points may be characterized by;

$$
\beta_{z, \alpha}=\gamma^{\alpha}=0, \bar{\beta}_{\bar{z}}^{\alpha}=\bar{\gamma}_{\alpha}=0
$$

for any $\alpha \in \Delta_{+}$. This is consistent with the quartet confinements (25).

On the fixed points (26) the residual dynamical degrees of freedom are $\phi, X$ and the $T^{c}$-ghosts $\zeta, \xi$. The action of these fields may be derived from the gauge fixed action $I^{g . f}$ (9) by dropping out the irrelevant terms (25). Introducing a complex boson $\varphi=\phi+i X$, it is given by;

$$
\begin{aligned}
& I_{\text {fixed point }}\left(\varphi, \varphi^{*}, \zeta, \bar{\zeta}, \xi, \bar{\xi}\right) \\
& =\frac{1}{4 \pi i} \int_{\Sigma} d^{2} z \partial_{z} \varphi \cdot \bar{\partial}_{\bar{z}} \varphi^{*}+\partial_{z} \varphi^{*} \cdot \bar{\partial}_{\bar{z}} \varphi+\frac{i}{2 \pi} \int_{\Sigma} \sqrt{2} \alpha_{-} \rho \cdot \varphi^{*} R \\
& \quad+\frac{1}{2 \pi i} \int_{\Sigma} d^{2} z \zeta_{z} \cdot \bar{\partial}_{\bar{z}} \xi+\bar{\zeta}_{\bar{z}} \cdot \partial_{z} \bar{\xi} .
\end{aligned}
$$

The action $I_{\text {fixed point }}$ is that of the Coulomb gas realization of the topological minimal model in the case of $n=2$, and its generalization for $n \geq 3$. In fact the system (27) is characterized by the following TCA;

$$
\begin{aligned}
G_{C . G}^{+} & =\sqrt{2} \alpha_{-} \rho \cdot \partial \xi+i \xi \cdot \partial \varphi^{*}, \\
G_{C . G}^{-} & =\sqrt{2} \alpha_{-} \rho \cdot \partial \zeta_{z}+i \zeta_{z} \cdot \partial \varphi, \\
T_{C . G} & =-\partial \varphi \cdot \partial \varphi^{*}+i \sqrt{2} \alpha_{-} \rho \cdot \partial^{2} \varphi^{*}-\zeta_{z} \cdot \partial \xi \\
J_{C . G} & =-i \sqrt{2} \alpha_{-} \rho \cdot \partial\left(\varphi-\varphi^{*}\right)+N_{\zeta \xi} .
\end{aligned}
$$


Modulo G/T-BRST exact terms this TCA is equal to that generated by $\left\{T_{T^{c}}, G_{T^{c}}^{ \pm}, J_{T^{c}}\right\}$ which describes the reduced system in the operator formulation. I Thus the TFT (27) is preceisely what we wish to have.

The TFT on the $G / T$-BRST fixed points (27) will play an important role in the study of the "WG-gravity" [13]. These will be studied in separate papers [7], [8]. Here we give some comments: In the case of $n=2$ the TFT on the fixed points is precisely the topological matter system in the K.Li's theory of 2 dimensional gravity [12. And this TFT is equivalent to "the abelian model" [1], that is, the topological $U(1)$ gauge theory coupled to 2 dimensional gravity. Since our main purpose in this article is to construct the TFT on the BRST fixed points, we neglect its coupling to 2 dimensional gravity. It is worth mentioning that the process of twisting $N=2$ symmetry in 2 dimension amounts to a mixing of Lorentz and internal $U(1)$ rotations and redefinition of the quantum number of the fields. This indicates [7] the possibility that the coupling to 2 dimensional topological gravity can be performed via the back-ground gauge field which we set trivial in (7).

Our discussion given in this article would carry over to the case of $G=S L(n, R)$. The $G / T$-BRST fixed points will be characterized by the same condition as (26), and the TFT on these fixed points is that described by the action (27) (with an appropriate change of $\alpha_{ \pm}$). It is known [14] that the $S L(2, R) / U(1)$ gauged WZW model (with a slightly different $U(1)$ embedding) describes a 2 dimensional target space geometry with a metrid

$$
d s^{2}=\frac{d u d v}{1-u v},
$$

which gives a 2 dimensional black hole. The curvature of the metric blows up at $u v=1$. Thus the space-time singularities appear at $u v=1$. This region of the singularity is the $S L(2, R) / U(1)$-BRST fixed points (26)]. Hence, as discussed in [2], the TFT on the fixed points will describe the region of the singularities in the black hole geometry.

\footnotetext{
${ }^{7}$ Because $H^{\text {total }}, \hat{H}(13)$ are equal to $i \alpha_{+} \partial \varphi^{*}, i \alpha_{+} \partial \phi$ respectively modulo $G / T$-BRST exact terms.

${ }^{8}$ We follow the notation in 14 .

${ }^{9}$ To be precise, the singularities and the fixed points are related by "duality" 15 .
} 


\section{Acknowledgements}

We would like to thank Prof.T.Eguchi for several discussions and continuous encouragement during this work was done.

\section{References}

[1] E.Witten,Nucl.Phys.B371(1992)191.

[2] T.Eguchi,Mod.Phys.Lett.A7(1992)85.

[3] T.Eguchi andS.K.Yang,Mod.Phys.Lett.A5(1990)1693.

[4] Y.Kazama andH.Suzuki,Nucl.Phys.B321(1989)232.

[5] K.Gawedzki andA.Kupiainen,Nucl.Phys.B320(1989)625.

[6] D.Karabali andH.J.Schnitzer,Nucl.Phys.B329(1990)649.

[7] T.Nakatsu andY.Sugawara,preprint UT-598.

[8] T.Nakatsu andY.Sugawara,preprint UT-600.

[9] K.Ito andY.Kazama,Mod.Phys.Lett.A5(1990)215.

[10] M.Bershadsky andH.Ooguri,Comm.Math.Phys.126(1989)49.

[11] J.M.Figueroa-O'Farill,Nucl.Phys.B343(1990)450;

N.Hayashi,Mod.Phys.Lett.A6(1991)885.

[12] K.Li,Nucl.Phys.B354(1991)711,725;

R.Dijkgraaf,E.Verlinde,andH.Verlinde,Nucl.Phys.B352(1991)59.

[13] J.-L.Gervais,andY.Matsuo,Phys.Lett.274B(1992)304;

G.Sotokov,M.Stanishikov,andC.J.Zhn,Nucl.Phys.B356(1991)245;

A.Gerashimov,A.Levin,andA.Marshakov,Nucl.Phys.B360(1991)95. 
[14] E.Witten,Phy.Rev.D44(1991)314.

[15] R.Dijkgraaf,E.Verlinde,andH.Verlinde,Nucl.Phys.B371(1992)267; A.Giveon,Mod.Phys.Lett.A6(1991)2843. 